\title{
Convergence of Multi-Grid Iterations Applied to Difference Equations
}

\author{
By Wolfgang Hackbusch
}

\begin{abstract}
Convergence proofs for the multi-grid iteration are known for the case of finite element equations and for the case of some difference schemes discretizing boundary value problems in a rectangular region. In the present paper we give criteria of convergence that apply to general difference schemes for boundary value problems in Lipschitzian regions. Furthermore, convergence is proved for the multi-grid algorithm with Gauss-Seidel's iteration as smoothing procedure.
\end{abstract}

1. Introduction. Systems of linear equations arising from boundary value problems can be solved very fast by the multi-grid iteration (cf. [1]-[6], [9], [11]). Although, the multi-grid algorithms are applied successfully to a general class of problems, the proofs of convergence are restricted to a very special class of problems. In the case of special finite element equations for boundary value problems with smooth boundaries proofs of convergence are given by Astrachancev [1] and Nicolaides [9] In [6] the author established general criteria and proved the convergence for general finite element problems.

The second important class of problems are systems of difference equations discretizing boundary value problems. The model problem of Poisson's equation in a rectangle (and similar problems) can be analyzed easily by means of Fourier transformation (cf. Fedorenko [4]). In the case of certain difference schemes for problems with variable coefficients and a rectangular region, Bachvalov [2] and Wesseling [11] proved the convergence of the multi-grid iteration. But two gaps are still to be filled. Convergence proofs are missing for the case of nonrectangular regions. Moreover, all proofs cited above require a special smoothing procedure (cf. Section 4) related to the Jacobi iteration. In practice smoothing by the Gauss-Seidel iteration is preferred (cf. [3], [5]). This paper contains general criteria that apply to difference schemes in general regions and to smoothing by Gauss-Seidel.

In Section 2 we describe the multi-grid algorithm very briefly. For further comments we refer, for instance, to [6]. As pointed out in [6] the convergence can be concluded from an 'approximation property' and a 'smoothing property'. The first one is studied in Section 3. A criterion is proved and its assumptions are verified in the case of a very general difference scheme. It turns out that the crux of the assumptions is a certain regularity condition (3.6b) that is proved in [7] for the case of Dirichlet

Received April 13, 1979.

AMS (MOS) subject classifications (1970). Primary 65F10, 65N20.

Key words and phrases. Multi-grid method, difference equations, boundary value problems. 
boundary values. The smoothing property is investigated in Section 4 , in particular, for the case of Gauss-Seidel's iteration as smoothing procedure.

2. Multi-Grid Iteration. Let

$$
h_{0}>h_{1}>\cdots>h_{l}>\cdots>0
$$

be a sequence of grid sizes. $l$ is called the 'level number'. The discretization of the continuous problem (boundary value problem)

$$
L u=f
$$

with step size $h_{l}$ is denoted by

$$
L_{l} u_{l}=f_{l} \quad(l \geqslant 0) .
$$

The solution $u_{l}$ of (2.3), as well as the right-hand side $f_{l}$, belongs to a finite-dimensional vector space $V_{l}$.

The system (2.3) of linear equations is to be solved by the multi-grid algorithm described below. It uses auxiliary equations of the form $L_{m} u_{m}=g_{m}$ for $m=0,1$, $\ldots, l-1$. The connection of grid functions of different levels is given by a prolongation

$$
p_{l, l-1}: V_{l-1} \rightarrow V_{l}
$$

and a restriction

$$
r_{l-1, l}: V_{l} \rightarrow V_{l-1}
$$

Since a detailed explanation of the multi-grid algorithm is contained in [5], [6], we give only a brief description by means of a program.

$$
\begin{aligned}
& \text { procedure } m g m(l, u, f) \text { : integer } l \text {; array } u, f \text {; } \\
& \text { if } l=0 \text { then } u:=L_{0}^{-1} * f_{0} \text { else } \\
& \text { begin integer } j \text {; array } v, d \text {; } \\
& \text { for } j:=1 \text { step } 1 \text { until } \nu \text { do } u:=G_{l}(u, f) \text { : } \\
& d:=r_{l-1, l} *\left(L_{l} * u-f\right) ; v:=0 \text {; } \\
& \text { for } j:=1 \text { step } 1 \text { until } \gamma \text { do } \operatorname{mgm}(l-1, v, d) \text {; } \\
& u:=u \rightarrow p_{l, l-1} * v
\end{aligned}
$$

end;

The meaning of the parameters is the following. $l \geqslant 0$ is the actual level number. $f \in$ $V_{l}$ is the right-hand side to the problem in consideration (e.g., $f=f_{l}$ in case of (2.3)). $u$ has an arbitrary input value $u_{l}^{(i)} \in V_{l}(i$ : number of iterations). The procedure mgm computes the next iterate $u=u_{l}^{(i+1)}$ as output. The procedure depends on the positive numbers $\nu$ (number of iterations of the smoothing procedure $G_{l}$ ) and $\gamma$ (number of $m g m$ iterations per level). The smoothing procedure is of the form

$$
G_{l}\left(v_{l}, f_{l}\right)=G_{l} v_{l}+H_{l} f_{l} \quad\left(v_{l}, f_{l} \in V_{l}\right) \text { with } G_{l}+H_{l} L_{l}=I .
$$

The convergence of the multi-grid algorithm depends on the choice of $\nu, \gamma$, on the coarsest step size $h_{0}$ and on the maximal ratio $\sup \left\{h_{l-1} / h_{l}: l \geqslant 1\right\}<\infty$. Usually, the last ratio is constant, e.g. equal to 2. In the following $\gamma=2$ is fixed (for $\gamma=1$ compare $[6$, Corollary 3.8$])$. 
We say that the multi-grid iteration 'converges' if it converges for a suitable choice of $h_{0}$ and $\nu$; more precisely if the iteration matrix $M_{l}=M_{l}\left(\nu, h_{0}, h_{1}, \ldots, h_{l}\right)$ [defined by $u_{l}^{(i+1)}-u_{l}=M_{l}\left(u_{l}^{(i)}-u_{l}\right), u_{l}=L_{l}^{-1} f_{l}$ ] satisfies

$$
\left\|M_{l}\right\| \leqslant C(\nu)<1 \text { for } \nu_{\min } \leqslant \nu \leqslant \nu_{\max }\left(h_{1}\right), l \geqslant 1,
$$

where $C(\nu) \rightarrow 0$ as $\nu \rightarrow \infty$ and $\nu_{\max }(h) \rightarrow \infty$ as $h \rightarrow 0$. The matrix norm $\|\cdot\|$ is associated with some suitable vector norm on $V_{l}$.

We recall the following result of [6]. Here and in the sequel $C$ denotes a generic constant independent of $l$.

Proposition 1. Let $\|\cdot\|_{1}$ and $\|\cdot\|_{2}$ be two suitable (not necessarily different) norms on $V_{l}(l \geqslant 0)$ and define the matrix norms $\|A\|_{i, j}(i, j=1,2)$ of $A: V_{l} \rightarrow V_{m}$ by $\sup \left\{\|A v\|_{j} /\|v\|_{i}: 0 \neq v \in V_{l}\right\}$. Assume the smoothing property

$$
\left\|L_{l} G_{l}^{\nu}\right\|_{2,1} \leqslant C_{0}(\nu) h_{l}^{-\alpha} \text { for all } l \geqslant 0,1 \leqslant \nu \leqslant \nu_{\max }\left(h_{1}\right)
$$

with $C_{0}(\nu) \rightarrow 0(\nu \rightarrow \infty), \nu_{\max }(h) \rightarrow \infty(h \rightarrow 0)$, and $G_{l}$ from $(2.4)$ for suitable $\alpha \geqslant 0$. Assume the approximation property

$$
\left\|L_{l}^{-1}-p_{l, l-1} L_{l-1}^{-1} r_{l-1, l}\right\|_{1,2} \leqslant C h_{l-1}^{\alpha} \quad \text { for all } l \geqslant 1
$$

with $\alpha$ from (2.6). Furthermore, the estimates

$$
\begin{gathered}
\frac{1}{C}\left\|v_{l-1}\right\|_{2} \leqslant\left\|p_{l, l-1} v_{l-1}\right\|_{2} \leqslant C\left\|v_{l-1}\right\|_{2} \quad \text { for all } v_{l-1} \in V_{l-1}, l \geqslant 1, \\
\left\|G_{l}^{\nu}\right\|_{2,2} \leqslant C \quad \text { for all } 1 \leqslant v \leqslant v_{\max }\left(h_{1}\right), l \geqslant 0 \\
h_{l}<h_{l-1} \leqslant C h_{l} \text { for all } l \geqslant 1
\end{gathered}
$$

are required. Then the multi-grid iteration with $\gamma=2$ converges: (2.5) holds with $\|\cdot\|=\|\cdot\|_{2,2}$.

\section{The Approximation Property.}

3.1. A Criterion Implying the Approximation Property. Assume

$$
r_{l-1, l} L_{l} p_{l, l-1}=L_{l-1}+\delta_{l-1} \quad(l \geqslant 1),
$$

where $\delta_{l-1}$ is small enough in the following sense:

$$
\left\|L_{l-1}^{-1} \delta_{l-1} r_{l-1, l}^{\prime} L_{l}^{-1}\right\|_{1,2} \leqslant C h_{l-1}^{\alpha} \quad(l \geqslant 1) .
$$

$r_{l-1, l}^{\prime}$ is a suitable restriction involved in (3.3) given below. If $L_{l}$ is the stiffness matrix of a finite element method, (3.1a) holds with $\delta_{l-1}=0$ (cf. [6]). $\delta_{l-1}$ vanishes, too, if $L_{l-1}$ is defined as in [5].

Moreover, we need the estimate

$$
\left\|L_{l-1}^{-1} r_{l-1, l} L_{l}\right\|_{2,2} \leqslant C \quad(l \geqslant 1)
$$

and the existence of some linear mapping $r_{l-1, l}^{\prime}: V_{l} \rightarrow V_{l-1}(l \geqslant 1)$ with

$$
\left\|\left[I-p_{l, l-1} r_{l-1, l}^{\prime}\right] L_{l}^{-1}\right\|_{1,2} \leqslant C h_{l-1}^{\alpha} \quad(l \geqslant 1) .
$$


$\alpha$ involved in (3.1b) and (3.3) is the exponent from (2.6).

Lemma 1. Assume that there are norms $\|\cdot\|_{0}$ and $\|\cdot\|_{3}$ on $V_{l}$ such that

$$
\left\|L_{l}^{-1}\right\|_{0,2} \leqslant C, \quad\left\|L_{l}^{-1}\right\|_{1,3} \leqslant C, \quad\left\|L_{l}\right\|_{2,0} \leqslant C \quad(l \geqslant 0) .
$$

Then (3.1a, b), (3.2), and (3.3) follow from $(3.4 \mathrm{~b}, \mathrm{c}, \mathrm{d})$ :

$$
\begin{gathered}
\left\|r_{l-1, l}\right\|_{0,0} \leqslant C, \quad\left\|r_{l-1, l}^{\prime}\right\|_{3,3} \leqslant C \quad(l \geqslant 1), \\
\left\|\delta_{l-1}\right\|_{3,0} \leqslant C h_{l-1}^{\alpha} \quad(l \geqslant 1) \\
\left\|I-p_{l, l-1} r_{l-1, l}^{\prime}\right\|_{3,2} \leqslant C h_{l-1}^{\alpha} \quad(l \geqslant 1) .
\end{gathered}
$$

(3.4d) describes the approximation of grid functions of $V_{l}$ by $p_{l, l-1} V_{l-1}$ : For all $v_{l} \in V_{l}$ there is $v_{l-1} \in V_{l-1}$ (namely $r_{l-1, l}^{\prime} v_{l}$ ) with $\left\|v_{l}-p_{l, l-1} v_{l-1}\right\|_{2} \leqslant$ $C h_{l-1}^{\alpha}\left\|v_{l}\right\|_{3}$. If $\alpha>0,\|\cdot\|_{3}$ must define a finer topology than $\|\cdot\|_{2}$.

In Proposition 1 the approximation property (2.7) may be replaced by (3.1)-(3.3):

CRITERION 1. (2.8), (3.1a, b), (3.2), and (3.3) imply the approximation property (2.7). By Lemma 1 also (2.8) and (3.4a-d) are sufficient.

Proof. Since $\left[I-p_{l, l-1} L_{l-1}^{-1} r_{l-1, l} L_{l}\right] p_{l, l-1}=-p_{l, l-1} L_{l-1}^{-1} \delta_{l-1}$ by (3.1a), it follows that

$$
\begin{gathered}
\left\|L_{l}^{-1}-p_{l, l-1} L_{l-1}^{-1} r_{l-1, l}\right\|_{1,2}=\left\|\left[I-p_{l, l-1} L_{l-1}^{-1} r_{l-1, l} L_{l}\right] L_{l}^{-1}\right\|_{1,2} \\
=\|\left[I-p_{l, l-1} L_{l-1}^{-1} r_{l-1, l} L_{l}\right]\left[I-p_{l, l-1} r_{l-1, l}^{\prime}\right] L_{l}^{-1} \\
\quad-p_{l, l-1} L_{l-1}^{-1} \delta_{l-1} r_{l-1, l}^{\prime} L_{l}^{-1} \|_{1,2} \\
\leqslant\left\{1+\left\|p_{l, l-1}\right\|_{2,2}\left\|L_{l-1}^{-1} r_{l-1, l} L_{l}\right\|_{2,2}\right\}\left\|\left[I-p_{l, l-1} r_{l-1, l}^{\prime}\right] L_{l}^{-1}\right\|_{1,2} \\
\quad+\left\|p_{l, l-1}\right\|_{2,2}\left\|L_{l-1}^{-1} \delta_{l-1} r_{l-1, l}^{\prime} L_{l}^{-1}\right\|_{1,2} .
\end{gathered}
$$

Hence, (2.8), (3.1b), and (3.3) yield (2.7). result:

Using $r_{l-1, l}\left[I-L_{l} p_{l, l-1} L_{l-1}^{-1} r_{l-1, l}\right]=-\delta_{l-1} L_{l-1}^{-1} r_{l-1, l}$, we obtain a similar

Criterion 2. Assume (3.1a),

$$
\begin{gathered}
\left\|r_{l-1, l}\right\|_{1,1} \leqslant C \quad(l \geqslant 1), \\
\left\|L_{l}^{-1} p_{l, l-1}^{\prime} \delta_{l-1} L_{l-1}^{-1}\right\|_{1,2} \leqslant C h_{l-1}^{\alpha} \quad(l \geqslant 1), \\
\left\|L_{l} p_{l, l-1} L_{l-1}^{-1}\right\|_{1,1} \leqslant C \quad(l \geqslant 1), \\
\left\|L_{l}^{-1}\left(I-p_{l, l-1}^{\prime} r_{l-1, l}\right)\right\|_{1,2} \leqslant C h_{l-1}^{\alpha} \quad(l \geqslant 1),
\end{gathered}
$$

for a suitable linear mapping $p_{l, l-1}^{\prime}: V_{l-1} \rightarrow V_{l}$. Then (2.7) follows.

3.2. Application of the Criterion. In the following we verify the conditions of Criterion 1 for the following example.

Example. Let $L_{l}(l \geqslant 0)$ be an elliptic difference operator of order $2 m$, i.e. the discretization of an elliptic differential operator of order $2 m$. Let $H_{0}^{s}$ be the space of 
all complex-valued grid functions defined on the $d$-dimensional grid $\Omega\left(h_{l}\right)=$ $\left\{x \in \Omega \subset \mathbf{R}^{d}: x / h_{l} \in \mathbf{Z}^{d}\right\}$ endowed with the norm

$$
\begin{aligned}
& |u|_{s}=(h / 2 \pi)^{d / 2}\left\|\left[1+h^{-2} \sum_{j=1}^{d} \sin ^{2}\left(\xi_{j} / 2\right)\right]^{s / 2} \sum_{x \in \Omega(h)} u(x) e^{i x \xi / h}\right\|_{1 L^{2}\left([-\pi, \pi]^{d}\right)} \\
& (s \geqslant 0) \\
& |u|_{-s}=\sup \left\{(h / 2 \pi)^{d}\left|\sum_{x \in \Omega(h)} u(x) \bar{v}(x)\right| /|v|_{s}: 0 \neq v \in H^{s}\right\} \quad\left(s \geqslant 0, h=h_{l}\right)
\end{aligned}
$$

(denoted by $|\cdot|_{s, 0}$ in [7]) corresponding to the norm of the Sobolev space $H_{0}^{s}(\Omega)$ if $s+1 / 2 \neq$ integer. We define the associated matrix norms by

$$
|A|_{s, t}=\sup \left\{|A u|_{t} /|u|_{s}: 0 \neq u \in H_{0}^{s}\right\} .
$$

Choose $\alpha,\|\cdot\|_{1}$, and $\|\cdot\|_{2}$ (and $\|\cdot\|_{0},\|\cdot\|_{3}$ of Lemma 1 ) by

$$
\begin{gathered}
\alpha=\theta+\theta^{\prime}, \quad\|\cdot\|_{1}=\left.1 \cdot\right|_{\theta-m}, \quad\|\cdot\|_{2}=\left.1 \cdot\right|_{m-\theta^{\prime}}, \\
\|\cdot\|_{0}=\left.1 \cdot\right|_{-m-\theta^{\prime}}, \quad\|\cdot\|_{3}=\left.1 \cdot\right|_{m+\theta}
\end{gathered}
$$

for some $\theta, \theta^{\prime} \in[0, m]$ with $\alpha=\theta+\theta^{\prime}>0$. The condition $\alpha>0$ will be important in Section 4.

The estimate

$$
\left|L_{l}\right|_{\vartheta+m, \vartheta-m} \leqslant C(\vartheta) \quad(l \geqslant 0, \vartheta \in \mathbf{R})
$$

holds if the coefficients of the difference scheme $L_{l}$ are sufficiently smooth (cf. Lemma 7). In [7] we proved

$$
\begin{aligned}
& \left|L_{l}^{-1}\right|_{\vartheta-m, \vartheta+m} \leqslant C \\
& \quad \text { for all } l \geqslant 0, \vartheta \in\left[-\theta_{0}^{\prime}, \theta_{0}\right]\left(\theta_{0}, \theta_{0}^{\prime} \in[0,1 / 2), \theta_{0}+\theta_{0}^{\prime}>0\right)
\end{aligned}
$$

under very weak assumptions. The main requirements are stability of $L_{l}$ with respect to $l_{2}=H_{0}^{0}$ and ellipticity of $L_{l}$. It suffices that the underlying region $\Omega$ is Lipschitzian. The assumption on the smoothness of the coefficients is very weak, too. (3.6b) holds even for some schemes with irregular discretizations near the boundary. Symmetry of positive definiteness of $L_{l}$ are not required.

At first we discuss the estimates $(3.4 \mathrm{a}-\mathrm{d})$ of Lemma 1.

Note 1. (3.5) and (3.6a, b) imply the estimates (3.4a) of Lemma 1 if $0 \leqslant \theta \leqslant$ $\theta_{0}$ and $0 \leqslant \theta^{\prime} \leqslant \theta_{0}^{\prime}$.

For the discussion of $(3.4 \mathrm{~b}, \mathrm{~d})$ we restrict our considerations to the case of $m=1$. Let $h_{l-1} / h_{l} \in \mathrm{Z}$ and define $p_{l, l-1}^{0}$ by

$$
\left(p_{l, l-1}^{0} u\right)(x)=\prod_{j=1}^{d} \max \left\{0,1-\left|x_{j}-y_{j}\right| / h_{l-1}\right\} \quad\left(x \in \Omega\left(h_{l}\right), y \in \Omega\left(h_{l-1}\right)\right),
$$

where $u \in V_{l-1}$ is the unit vector with $u(y)=1, u(z)=0$ for $z \neq y . \quad p_{l, l-1}^{0}$ is an example of an interpolation of order 2. Furthermore, define $r_{l-1, l}^{0}$ as the mapping adjoint to $p_{l, l-1}^{0}:\left(u, p_{l, l-1}^{0} v\right)=\left(r_{l-1, l}^{0} u, v\right)$, where $(v, w)=h^{d} \Sigma_{x \in \Omega(h)} v(x) \bar{w}(x)$ with $h=h_{l}$ or $h_{l-1}$, respectively. In the usual case of $d=2$ and $h_{l-1}=2 h_{l}$, the mappings 
$p_{l, l-1}^{0}$ and $r_{l-1, l}^{0}$ become

$$
\begin{aligned}
&\left(p_{l, l-1}^{0} u\right)(x)=\left\{\begin{aligned}
& u(x) \quad \text { if } x \in \Omega\left(h_{l-1}\right), \\
& \frac{1}{2}\left[u\left(x+e_{j} h_{l}\right)+u\left(x-e_{j} h_{l}\right)\right] \text { if } x+e_{j} h_{l} \in \Omega\left(h_{l-1}\right) \\
& \frac{1}{4} \sum_{j, k=1,2} u\left(x+(-1)^{k} e_{1} h_{l}+(-1)^{j} e_{2} h_{l}\right) \quad \text { or } x-e_{j} h_{l} \in \Omega\left(h_{l-1}\right),
\end{aligned}\right. \\
&\left(r_{l-1, l}^{0} u\right)(x)= \frac{1}{4} u(x)+\frac{1}{8} \sum_{j, k=1,2} u\left(x+(-1)^{k} e_{j} h_{l}\right)
\end{aligned}
$$

where $e_{j}(j=1,2)$ are the unit vectors $(1,0),(0,1)$. Note that $u(y)=0$ if $y \notin$ $\Omega\left(h_{l}\right)$.

Note 2. Let $m=1 . p_{l, l-1}^{0}$ and $r_{l-1, l}^{0}$ defined above satisfy $(3.7 \mathrm{a}, \mathrm{b})$ :

$$
\left|p_{l, l-1}^{0}\right|_{s, s} \leqslant C, \quad\left|r_{l-1, l}^{0}\right|_{s, s} \leqslant C \quad \text { for all } l \geqslant 1,|s| \leqslant 2,
$$

(3.7b) $\left|I-p_{l, l-1}^{0} r_{l-1, l}^{0}\right|_{s, t} \leqslant C h_{l-1}^{s-t}$ for all $l \geqslant 1,-2 \leqslant t \leqslant s \leqslant 2, s-t \leqslant 2$.

COROllary 1 to Note 2. Assume $m=1$ and (3.5) and set $r_{l-1, l}^{\prime}=r_{l-1, l}^{0}$. Then (2.8) and the estimates (3.4b, d) of Lemma 1 hold for $p_{l, l-1}=p_{l, l-1}^{0}$ with $\alpha=\theta+\theta^{\prime}>0$. Moreover, (2.8) and (3.4d) remain valid if the coefficients of $p_{l, l-1}$ and $p_{l, l-1}^{0}$ differ by $O\left(h_{l}^{1+\theta}\right)$ and/or if the coefficients of $p_{l, l-1}$ and $p_{l, l-1}^{0}$ differ by $O(1)$ at points near the boundary (i.e., distance $\left.(x, \partial \Omega) \leqslant C h_{l}\right)$. Similarly, $(3.4 \mathrm{~b})$ remains true if the coefficients of $r_{l-1, l}$ and $r_{l-1, l}^{0}$ differ by $O\left(h^{1+\max \left(\theta_{,} \theta^{\prime}\right)}\right)$ or by $O$ (1) near the boundary.

Example. $p_{l, l-1}$ and $r_{l-1, l}$ defined in [5, Eq. (3.4)] satisfy (3.7a, b).

Corollary 2 to Note 2. Generalizations to $m>1$ are obvious. $p_{l, l-1}^{0}$ must be defined by interpolation of order $>m$.

Note that this requirement is weaker than the requirement "order of interpolation $\geqslant 2 m$ " of Brandt [3, p. 377].

Since $L_{l}$ and $L_{l-1}$ should be consistent discretizations of the same differential operator (2.2), the difference $\delta_{l-1}=r_{l-1, l} L_{l} p_{l, l-1}-L_{l-1}$ is expected to consist of terms of the following form:

$$
\begin{gathered}
\delta_{l-1}=\sum_{\beta, \beta^{\prime} \in \mathbf{Z}^{d},|\beta|+\left|\beta^{\prime}\right| \leqslant 2 m+1} \sum_{\gamma \in \mathbf{Z}^{d}} T^{\gamma} \partial^{\beta} d_{\gamma, \beta, \beta^{\prime}, l-1}(x, h) \partial^{\beta^{\prime}}, \\
\quad \sup \left\{\left|d_{\gamma, \beta, \beta^{\prime}, l-1}(x, h)\right|: x \in \Omega(h), l \geqslant 1\right\} \leqslant C h^{\vartheta}, \\
\vartheta= \begin{cases}1 & \text { if }|\beta|+\left|\beta^{\prime}\right|=2 m+1,|\beta|,\left|\beta^{\prime}\right| \leqslant m+1, \\
\theta+\theta^{\prime} & \text { if }|\beta|+\left|\beta^{\prime}\right| \leqslant 2 m,|\beta|,\left|\beta^{\prime}\right| \leqslant m,\end{cases}
\end{gathered}
$$


where $\beta, \beta^{\prime}$, and $\gamma$ are multi-indices with $|\beta|=\beta_{1}+\cdots+\beta_{d}, \partial^{\beta}=\partial_{1}^{\beta} \cdots \partial_{d}^{\beta}$, $\left(\partial_{j} u\right)(x)=\left[u(x)-u\left(x-e_{j} h\right] / h,\left(T^{\gamma} u\right)(x)=u(x+\gamma h), h=h_{l-1}\right.$ (cf. [7]). The definition of $\partial$ and $T$ makes sense since $u(x)$ is extended by zero outside $\Omega(h)$. (3.8b) may be replaced by other conditions involving Hölder continuity of $d_{\gamma, \beta, \beta^{\prime}, l-1}(\cdot, h)$.

Example. Consider the differential operator $L=-\left(a\left(x_{1}\right) u_{x_{1}}\right)_{x_{1}}-$ $\left(b\left(x_{2}\right) u_{x_{2}}\right)_{x_{2}}$. Discretize $\left(a u_{x_{1}}\right)_{x_{1}}$ by $L_{l}^{\mathrm{I}} u=-h^{-2}\left[-a^{+} u^{+}+\left(a^{+}+a^{-}\right) u-a^{-} u^{-}\right]$ with $u=u(x), u^{ \pm}=u\left(x_{1} \pm h, x_{2}\right), a^{ \pm}=a\left(x_{1} \pm h / 2\right)$. Similarly, $L_{l}^{\text {II }}$ is the discretization of the second term of $L . L_{l}$ is the sum $L_{l}^{\mathrm{I}}+L_{l}^{\mathrm{II}}$ with $h=h_{l}$. Let $p_{l, l-1}=p_{l, l-1}^{0}$ and $r_{l-1, l}=r_{l-1, l}^{0}$ as defined above. Then $\delta_{l-1}^{\mathrm{I}}=r_{l-1, l} L_{l}^{\mathrm{I}} p_{l, l-1}-L_{l-1}^{\mathrm{I}}$ becomes $T_{1} \partial_{1}\left[a\left(x_{1}-h / 2\right)-1 / 2 a\left(x_{1}-3 h / 4\right)-1 / 2 a\left(x_{1}-h / 4\right)\right] \partial_{1}$

$+T_{1} T_{2} \partial_{2}^{2}\left[-h^{2}\left(a\left(x_{1}-h / 4\right)+a\left(x_{1}-3 h / 4\right)\right) / 16\right] \partial_{1}^{2}$

$+T_{2} \partial_{2}^{2}\left[-h\left(a\left(x_{1}+3 h / 4\right)+a\left(x_{1}+h / 4\right)-a\left(x_{1}-h / 4\right)-a\left(x_{1}-3 h / 4\right)\right) / 16\right] \partial_{1}$, where $\left(T_{1} u\right)(x)=u\left(x_{1}+h, x_{2}\right),\left(T_{2} u\right)(x)=u\left(x_{1}, x_{2}+h\right)$ and $h=h_{l-1}$. The brackets contain the coefficients of (3.8a). Obviously, (3.8b) holds if $a(\cdot)$ is Hölder continuous with exponent $\theta+\theta^{\prime}=\alpha<1$. If $a(\cdot)$ has Lipschitz continuous derivatives, (3.8b) holds with $\theta=\theta^{\prime}=1$.

Note 3. Let $\alpha$ and the norms be chosen according to (3.5). (3.8a, b) implies the estimate (3.4c) of Lemma 1. (3.4c) holds even if $\delta_{l-1}$ contains a further term of order $O\left(h_{l-1}^{-2 m}\right)$ at points near the boundary.

Proof. Use $\left|\partial^{\beta} u\right|_{0} \leqslant C|u|_{m+\theta}$ if $|\beta| \leqslant m+\theta$ and $h\left|\partial^{\beta} u\right|_{0} \leqslant C h^{\theta}|u|_{m+\theta}$ if $m+\theta \leqslant|\beta|=m+1$. For perturbations near the boundary apply the following lemma (cf. [7]).

Lemma 2. Let $\Omega(h)$ have 'property C' defined in [7]. Assume that the subset $\Gamma(h) \subset \Omega(h)$ satisfy distance $\left(x, h \mathrm{Z}^{d} \backslash \Omega(h)\right) \leqslant C h$ for some $C \neq C(h)$ and all $x \in$ $\Gamma(h)$, that means, all points of $\Gamma(h)$ have a distance less than $C h$ from the boundary. Define the restriction $\gamma$ by $(\gamma u)(x)=u(x)$ if $x \in \Gamma(h),(\gamma u)(x)=0$ otherwise. Then $|\gamma|_{s, t} \leqslant C^{\prime} h^{s-t}$ is valid.

A sufficient condition for 'property $\mathrm{C}^{\prime}$ ' is that $\Omega$ is Lipschitzian.

From Notes 1-3, Lemma 1 and Criterion 1 one concludes that the approximation property (2.7) holds for a very general class of difference schemes $L_{l}$.

Example (Application to the Shortley-Weller scheme). Discretize $-\Delta u=f$ (in a Lipschitz region $\Omega \subset \mathbf{R}^{2}$ ), $u=g$ (on $\partial \Omega$ ) by the Shortley-Weller scheme $L_{l}$ (cf. [5] , [8, p. 203ff.]). In [7, Note 2.3] we proved (3.6b) with $\theta_{0}^{\prime}=0, \theta_{0}>0$. But note that (3.6a) is not valid since the diagonal $D_{l}$ of the matrix $L_{l}$ can be arbitrarily large. Nevertheless, $\left(h_{l}^{2} D_{l}\right)^{-1} L_{l}$ and $L_{l}\left(h_{l}^{2} D_{l}\right)^{-1}: H_{0}^{\theta+m} \rightarrow H_{0}^{\theta-m}$ are uniformly bounded.

Define $\theta^{\prime}=0, p_{l, l-1}=p_{l, l-1}^{0}, r_{l-1, l}=r_{l-1, l}^{0}\left(h_{l}^{2} D_{l}\right)^{-1}, r_{l-1, l}^{\prime}=$ $\left(h_{l-1}^{2} D_{l-1}\right)^{-1} r_{l-1, l}^{0}$ (or define $p_{l, l-1}$ and $r_{l-1, l}$ as in [5]). Then (3.1a, b), (3.2), and (3.3) are fulfilled. For a proof modify Lemma 1: Split $r_{l-1, l} L_{l}$ into $r_{l-1, l}^{0} \dot{ }$. $\left[\left(h_{l}^{2} D_{l}\right)^{-1} L_{l}\right]$ and $\delta_{l-1} r_{l-1}^{\prime}$, into $\left[\delta_{l-1}\left(h_{l-1}^{2} D_{l-1}\right)^{-1}\right] \cdot r_{l-1, l}^{0}$. Thus, we have shown the approximation property (2.7) with $\alpha=\theta>0$ by means of Criterion 1 . 


\section{Criteria Implying the Smoothing Property.}

4.1. Preparing Lemmata. The following lemma describes a norm equivalent to $\left.1 \cdot\right|_{s}$.

Lemma 3. Let $\Omega(h)$ have 'property C' (cf. Lemma 2). Assume $L_{l, 0}$ to be a positive definite and $H_{0}^{m}$-elliptic difference operator of order $2 m$, i.e., $L_{l, 0}=L_{l, 0}^{*}$ and $|u|_{m}^{2} / C \leqslant\left(L_{l, 0} u, u\right) \leqslant C|u|_{m}^{2}$, where $(u, v)=h^{d} \Sigma_{x \in \Omega(h)} u(x) \bar{v}(x)$. The fractional powers of $\Lambda:=\left(L_{l, 0}\right)^{1 /(2 m)}$ are well defined. Then $|u|_{s}$ and $\left|\Lambda^{s} u\right|_{0}$ are equivalent: $\left(1 / C^{\prime}\right)|u|_{s} \leqslant\left|\Lambda^{s} u\right|_{0} \leqslant C^{\prime}|u|_{s}$, for $-m \leqslant s \leqslant m$. $C^{\prime}$ does not depend on $h_{l}$.

Proof. Use Lemma 2.1 of [7] and the following lemma.

Lemma 4 (Inter polation). Let $H_{1}$ and $H_{2}$ be two Hilbert spaces. $A: H_{1} \rightarrow$ $H_{2}, \Lambda_{i}: H_{i} \rightarrow H_{i}$ and $\Lambda_{i}^{-1}: H_{i} \rightarrow H_{i}(i=1,2)$ are assumed to be bounded. Furthermore, let $\Lambda_{1}$ and $\Lambda_{2}$ be positive definite. Then the inequality

$$
\left\|\Lambda_{2}^{\gamma} A \Lambda_{1}^{-\gamma}\right\|_{H_{1} \rightarrow H_{2}} \leqslant C_{1}^{\left(\gamma_{2}-\gamma\right) /\left(\gamma_{2}-\gamma_{1}\right)} C_{2}^{\left(\gamma-\gamma_{1}\right) /\left(\gamma_{2}-\gamma_{1}\right)}
$$

holds for all $\gamma \in\left[\gamma_{1}, \gamma_{2}\right]$ if it is valid for $\gamma=\gamma_{1}$ and $\gamma=\gamma_{2}$.

Proof. Set $\varphi(\gamma)=\left\|\Lambda_{2}^{\gamma} A \Lambda_{1}^{-\gamma}\right\|_{H_{1} \rightarrow H_{2}}$ and note that

$$
\varphi(\gamma)^{2}=\left\|\Lambda_{2} A \Lambda_{1}^{-2 \gamma} A^{*} \Lambda_{2}^{\gamma}\right\|_{H_{2} \rightarrow H_{2}}=\rho\left(\Lambda_{2}^{\gamma^{\prime}} A \Lambda_{1}^{-\gamma^{\prime}} \Lambda_{1}^{-\gamma^{\prime \prime}} A^{*} \Lambda_{2}^{\gamma^{\prime \prime}}\right) \leqslant \varphi\left(\gamma^{\prime}\right) \varphi\left(\gamma^{\prime \prime}\right)
$$

for all $\gamma^{\prime}, \gamma^{\prime \prime}$ with $\gamma^{\prime}+\gamma^{\prime \prime}=\gamma$ ( $\rho$ : spectral radius). Therefore, the estimate follows by bisection for all $\gamma=\gamma_{1}+\nu 2^{-\mu}\left(\gamma_{2}-\gamma_{1}\right)$ with $\nu, \mu \in Z, \mu \geqslant 0,0 \leqslant \nu \leqslant 2^{\mu}$. The continuity of $\varphi(\gamma)$ concludes the proof.

The preceding lemmata yield the following estimates.

Lemma 5. The estimates $(4,1 \mathrm{a}, \mathrm{b}, \mathrm{c})$ hold with $C$ independent of $h_{l}$ :

$$
|A|_{r, r} \leqslant C|A|_{s, s}^{(t-r) /(t-s)}|A|_{t, t}^{(r-s) /(t-s)} \quad(-m \leqslant s \leqslant r \leqslant t \leqslant m),
$$

$$
\begin{aligned}
& |A|_{r,-r} \leqslant C|A|_{0,0}^{(t-r) / t}|A|_{t,-t}^{r / t} \quad(0 \leqslant r \leqslant t \leqslant m \text { or } 0 \geqslant r \geqslant t \geqslant-m), \\
& |A|_{r,-s} \leqslant C|A|_{0,0}^{(2 m-r-s) /(2 m)}|A|_{2 m, 0}^{r /(2 m)}|A|_{0,-2 m}^{s /(2 m)} \\
& \quad(r \geqslant 0, s \geqslant 0, r+s \leqslant 2 m) .
\end{aligned}
$$

Proof. By virtue of Lemma 3, $|u|_{s}$ can be replaced with $\left|\Lambda^{s} u\right|_{0}$. Hence, $|A|_{r, s}$ becomes $\left|\Lambda^{s} A \Lambda^{-r}\right|_{0,0}$. Applying Lemma 4 with $\Lambda_{1}=\Lambda_{2}=\Lambda$ we obtain (4.1a).

(4.1b) follows by choosing $\Lambda_{1}=\Lambda, \Lambda_{2}=\Lambda^{-1}$. For the proof of (4.1c) apply Lemma 3 and (4.1a) with $2 m$ instead of $m$. We abbreviate $|A|_{p, q}$ by $a(p, q)$. Lemma 4 with $\Lambda_{1}=I, \Lambda_{2}=\Lambda$ yields $a(r+s, 0) \leqslant C a(0,0)^{1-\beta} a(2 m, 0)^{\beta}$ with $\beta=(r+s) /(2 m)$. Similarly, $a(0,-r-s) \leqslant C a(0,0)^{1-\beta} a(0,-2 m)^{\beta}$ follows. Applying (4.1a) to $\Lambda^{-r-s} A$ instead of $A$, one obtains $a(r,-s) \leqslant C a(r+s, 0)^{r /(r+s)} a(0,-r-s)^{s /(r+s)}$. Inserting the estimates of $a(r+s, 0)$ and $a(0,-r-s)$ we are led to $(4.1 \mathrm{c})$.

Smoothing by Gauss-Seidel's iteration is expressed by

$$
G_{l}\left(v_{l}, f_{l}\right)=\left(D_{l}-R_{l}\right)^{-1}\left(S_{l} v_{l}+f_{l}\right), \quad G_{l}=\left(D_{l}-R_{l}\right)^{-1} S_{l},
$$


where

$$
L_{l}=D_{l}-R_{l}-S_{l}
$$

Definition 1. The splitting (4.2b) is called 2-cyclic (cf. [10, p. 39]) if there are two distinct subsets $\Omega_{1}(h)$ and $\Omega_{2}(h)$ of $\Omega(h)$ with $\Omega_{1}(h) \cup \Omega_{2}(h)=\Omega(h)$ such that

$$
D_{l}=\omega_{1} L_{l} \omega_{1}+\omega_{2} L_{l} \omega_{2}, \quad R_{l}=-\omega_{2} L_{l} \omega_{1}, \quad S_{l}=-\omega_{1} L_{l} \omega_{2},
$$

where the restrictions $\omega_{j}$ are defined by $\left(\omega_{j} u\right)(x)=u(x)$ if $x \in \Omega_{j}(h)$ and $\left(\omega_{j} u\right)(x)$ $=0$ otherwise.

Throughout this section we shall assume

(4.3) $\Omega(h)$ have 'property C' (cf. Lemma 2); $\alpha,\|\cdot\|_{1},\|\cdot\|_{2}$ be defined by (3.5).

LEMma 6. Let the splitting (4.2b) be 2-cyclic and assume $L_{l}=L_{l}^{*}$ to be positive definite. Then $\left|L_{l} G_{l}^{\nu}\right|_{0,0} \leqslant\left|D_{l}\right|_{0,0} /(\nu+1 / 2)$ holds for all $\nu \geqslant 1$.

Proof. Numbering first the grid points of $\Omega_{1}(h)$ yields the following block structure:

$$
\begin{gathered}
L_{l}=\left[\begin{array}{cc}
d_{1} & -s \\
-r & d_{2}
\end{array}\right], \quad G_{l}=\left[\begin{array}{cc}
0 & d_{1}^{-1} s \\
0 & d_{2}^{-1} r d_{1}^{-1} s
\end{array}\right], \\
L_{l} G_{l}^{\nu}=\left[\begin{array}{cc}
0 & s\left\{\left[d_{2}^{-1} r d_{1}^{-1} s\right]^{\nu-1}-\left[d_{2}^{-1} r d_{1}^{-1} s\right]^{\nu}\right\} \\
0 & 0
\end{array}\right] .
\end{gathered}
$$

Hence, $\left|D_{l}^{-1 / 2} L_{l} G_{l}^{\nu} D_{l}^{-1 / 2}\right|_{0,0}^{2}=\left|\left[\begin{array}{cc}0 & 0 \\ 0\end{array}\right]\right|_{0,0}$ follows from $s^{*}=r$ with $A=$ $B^{2 \nu-1}(I-B)^{2}$ and $B=B^{*}=d_{2}^{-1 / 2} r d_{1}^{-1} s d_{2}^{-1 / 2}$. It is well known that $\rho\left(G_{l}\right)=\rho(B)$ $=\|B\| \leqslant 1$ (cf. Note 5 ), where $\|\cdot\|$ denotes the $|\cdot|_{0,0}$-norm restricted to the last block.

$$
\begin{aligned}
\|A\| & =\rho(A)=\sup \left\{\left|\lambda^{2 \nu-1}(1-\lambda)^{2}\right|: \lambda \in \text { spectrum of } B\right\} \\
& \leqslant \sup \left\{\lambda^{2 \nu-1}(1-\lambda)^{2}: 0 \leqslant \lambda \leqslant 1\right\} \leqslant 1 /(\nu+1 / 2)^{2}
\end{aligned}
$$

implies $\left|L_{l} G_{l}^{\nu}\right|_{0,0} \leqslant\left|D_{l}^{1 / 2}\right|_{0,0}^{2} H A \|^{1 / 2} \leqslant\left|D_{l}\right|_{0,0} /(\nu+1 / 2)$.

\subsection{General Criteria.}

Criterion 3. Assume (4.3), $\theta=\theta^{\prime}$, (3.6a) for $\vartheta=0$, and

$$
\begin{gathered}
\left|L_{l} G_{l}^{y}\right|_{0,0} \leqslant h_{l}^{-2 m} C(\nu) \quad \text { for } 1 \leqslant \nu \leqslant \nu_{\max }\left(h_{1}\right), l \geqslant 0 ; \\
C(\nu) \rightarrow 0(\nu \rightarrow \infty), \quad \nu_{\max }(h) \rightarrow \infty(h \rightarrow 0), \\
\left|G_{l}^{\nu}\right|_{m, m} \leqslant C \quad \text { for all } 1 \leqslant \nu \leqslant \nu_{\max }\left(h_{1}\right), l \geqslant 0 .
\end{gathered}
$$

Then the smoothing property (2.6) holds with $C_{0}(\nu)=C^{\prime}[C(\nu)]^{\theta / m}$.

Proof. (3.6a) $(\vartheta=0)$ and (4.4b) yield $\left|L_{l} G_{l}^{\nu}\right|_{m,-m} \leqslant\left|L_{l}\right|_{m,-m}\left|G_{l}^{\nu}\right|_{m, m} \leqslant{ }^{\circ} C$.

Hence, (4.1b) $(t=m, r=m-\theta)$ implies (2.6).

The following criterion applies also to the case of $\theta \neq \theta^{\prime}$ : 
Criterion 3*. Assume (4.3), (4.4a) and

$$
\left|L_{l}\right|_{2 m, 0} \leqslant C, \quad\left|L_{l}^{*}\right|_{2 m, 0} \leqslant C, \quad\left|G_{l}^{\nu}\right|_{0,0} \leqslant C,
$$

$$
\left|\tilde{G}_{l}^{\nu}\right|_{0,0} \leqslant C \quad\left(0 \leqslant \nu \leqslant \nu_{\max }\left(h_{1}\right), l \geqslant 0\right)
$$

where $\widetilde{G}_{l}=L_{l} G_{l} L_{l}^{-1}$. Then the smoothing property (2.6) holds with $C_{0}(\nu)=C^{\prime}$. $C(\nu)^{\alpha /(2 m)}\left[\alpha=\theta+\theta^{\prime}, c f .(3.5)\right]$.

Proof. (4.4b*) implies $\left|L_{l} G_{l}^{\nu}\right|_{2 m, 0}=\left|\widetilde{G}_{l}^{\nu} L_{l}\right|_{2 m, 0} \leqslant\left|\widetilde{G}_{l}^{\nu}\right|_{0,0}\left|L_{l}\right|_{2 m, 0} \leqslant C$. Since $\left|L_{l}^{*}\right|_{2 m, 0}=\left|L_{l}\right|_{0,-2 m}$, also $\left|L_{l} G_{l}\right|_{0,-2 m} \leqslant\left|L_{l}\right|_{0,-2 m}\left|G_{l}^{\nu}\right|_{0,0} \leqslant C$ holds. (4.1c) yields (2.6).

First we shall verify the conditions of Criteria 3 and $3^{*}$ for positive definite schemes. In a second step it is shown that additional terms of lower order may be added. Hence, all difference schemes with a hermitian principle part satisfy the smoothing property. In a third step we treat perturbations of order $O\left(h_{l}^{-2 m}\right)$ located at points near the boundary. Such perturbations often arise from special discretizations at the boundary.

Usually, the function $C_{0}(\nu)$ of $(2.6)$ is $C /(\nu+1)^{\alpha /(2 m)}$. Therefore, $C_{0}(\nu) \rightarrow 0$ requires $\alpha=\theta+\theta^{\prime}>0$. The choice of $\theta=\theta^{\prime}=0$ is excluded. The upper bound $\nu_{\max }(h)$ of $\nu$ in (2.6) may be omitted (i.e. $\nu_{\max }=\infty$ ) if $L_{l}$ is positive definite. In the case of other schemes $\nu_{\max }(h)$ might become finite (but $\nu_{\max }(h) \rightarrow \infty$ as $h \rightarrow 0$ ).

4.3. Case of Positive Definite Difference Schemes. Throughout this subsection we assume

$$
L_{l}=L_{l}^{*}, \quad \frac{1}{C}|u|_{m} \leqslant\left(L_{l} u, u\right) \leqslant C|u|_{m}
$$

as in Lemma 3. The proofs of convergence in [1], [2], [4], [6], [9], [11] require smoothing by

$$
G_{l}\left(v_{l}, f_{l}\right)=v_{l}-\omega_{l} h_{l}^{2 m}\left(L_{l} v_{l}-f_{l}\right), \quad G_{l}=I-\omega_{l} h_{l}^{2 m} L_{l} .
$$

If the diagonal of $L_{l}$ is a multiple of $I, G_{l}$ corresponds to a damped Jacobi iteration.

Note 4 (Smoothing by Jacobi Iteration). Assume (4.3), (4.5), (4.6) and $0 \leqslant$ $\omega_{l} \leqslant h_{l}^{-2 m} /\left[\left.L_{l}\right|_{0,0}\right.$. Then the smoothing property (2.6) holds for all $\nu\left(\nu_{\max }=\infty\right)$ with

$$
C_{0}(\nu)=C /(\nu+1 / 2)^{\alpha /(2 m)} \quad\left(\alpha=\theta+\theta^{\prime} \text { from }(3.5)\right) .
$$

Proof. One may choose $L_{l, 0}=L_{l}$ in Lemma 3. Thus, it suffices to estimate $A=\Lambda^{\theta-m} L_{l} G_{l}^{\nu} \Lambda^{\theta^{\prime}-m}=L_{l}^{\beta}\left(I-\omega_{l} h_{l}^{2 m} L_{l}\right)^{\nu}, \beta=\alpha /(2 m)$, with respect to $|\cdot|_{0,0}$. But this norm is equal to the spectral radius. Since the spectrum of $L_{l}$ is contained in $\left[0,1 /\left(\omega_{l} h_{l}^{2 m}\right)\right]$,

$$
\rho(A)=\sup \left\{\lambda^{\beta}\left(1-\omega_{l} h_{l}^{2 m} \lambda\right)^{\nu}: 0 \leqslant \omega_{l} h_{l}^{2 m} \leqslant 1\right\} \leqslant C /(\nu+1)^{\beta}
$$

proves Note 4.

The techniques of the following subsections can be applied to smoothing by (4.6), too. But since we are mainly interested in smoothing by Gauss-Seidel's iteration, henceforward our considerations are restricted to this subject. 
Note 5 (Smoothing by Gauss-Seidel). Assume (4.3), $\theta=\theta^{\prime}$, (4.5). Let $G_{l}$ be defined by $(4.2 \mathrm{a}, \mathrm{b})$, where the splitting $(4.2 \mathrm{~b})$ is required to be 2-cyclic. Then the smoothing property (2.6) holds with $C_{0}(\nu)$ from $(4.7)$ for all $\nu\left(\nu_{\max }(h)=\infty\right)$.

Proof. Since (4.2b) is 2-cyclic and $L_{l}$ is positive definite, $D_{l}$ is positive definite, too. Thus, the theorem of Ostrowski (cf. [8, p. 297], [10, p. 77]) applies resulting in $\left|L_{l}^{1 / 2} G_{l}^{\nu} L_{l}^{-1 / 2}\right|_{0,0} \leqslant 1(\nu \geqslant 0)$. By Lemma $3(4.4 \mathrm{~b})$ follows. Lemma 6 implies (4.4a) with $C(\nu)=C /(\nu+1 / 2)$ since $\left|D_{l}\right|_{0,0} \leqslant\left|L_{l}\right|_{0,0} \leqslant C h_{l}^{-2 m}$ results from (4.5) and $|u|_{m} \leqslant C h_{l}^{-m}|u|_{0}$. (3.6a) with $\vartheta=0$ holds by virtue of (4.5). Hence, all conditions of Criterion 3 are satisfied.

Example. Let $L_{l} u=f$ be the discretization of $-\operatorname{div}\left[\left(a\left(x_{1}\right), b\left(x_{2}\right)\right)^{T} \operatorname{grad} u\right]=\varphi$ in $\Omega$ and $u=0$ on $\partial \Omega$ as in the example of Section 3.1. (4.5) holds if $a\left(x_{1}\right), b\left(x_{2}\right)$ $\in[\epsilon, C] \subset(0, \infty)$. Use the 'red-black' ordering of the grid points: $\Omega_{1}(h)=\{x \in$ $\Omega(h):\left(x_{1}+x_{2}\right) / h$ even $\}$. If, in addition, $\Omega$ is a Lipschitz region, all conditions of Note 5 are satisfied. The smoothing property holds for all $\theta=\theta^{\prime}=\alpha / 2 \in(0, m]$.

Note 5 illustrates the application of Criterion 3. In order to apply Criterion $3^{*}$ the following lemmata give conditions implying $\left(4.4 \mathrm{~b}^{*}\right)$.

LEMma 7. The inequalities $\left|L_{l}\right|_{2 m, 0} \leqslant C$ and $\left|L_{l}^{*}\right|_{2 m, 0} \leqslant C$ hold if the coefficients are sufficiently smooth. More precisely, the estimates hold if $L_{l}$ is a finite sum of terms of the form

$$
T^{\gamma} \partial^{\beta} c\left(x, h_{l}\right) \partial^{\beta^{\prime}} \quad\left(\gamma, \beta, \beta^{\prime} \in \mathbf{Z}^{d}, \beta_{j} \geqslant 0, \beta_{j}^{\prime} \geqslant 0,|\beta|+\left|\beta^{\prime}\right| \leqslant 2 m\right),
$$

where all kth derivatives of $c\left(x, h_{l}\right)$ with respect to $x$ are uniformly Lipschitz continuous on $\bar{\Omega}$ for $k=\max \left(|\beta|,\left|\beta^{\prime}\right|\right)-1\left[\right.$ for $T^{\gamma}$ and $\partial^{\beta}$ compare Section 3, (3.8a)].

Proof. $\left|L_{l}\right|_{2 m, 0} \leqslant C$ requires $k \geqslant|\beta|-1$. Since $L_{l}^{*}$ contains $(-1)^{|\beta|+\left|\beta^{\prime}\right|} T^{\beta^{\prime}} \partial^{\beta^{\prime}} c \partial^{\beta} T^{\gamma+\beta}$, also $k \geqslant\left|\beta^{\prime}\right|-1$ must hold.

Lemma 8. The estimates $\left|G_{l}^{\nu}\right|_{0,0} \leqslant C,\left|\widetilde{G}_{l}^{\nu}\right|_{0,0} \leqslant C$ are valid for all $\nu \geqslant 0$ and $l \geqslant 0$ if the splitting (4.2b) is 2 -cyclic and if one of the following conditions holds:

$$
L_{l} \text { satisfies (4.5), }\left|D_{l}^{-1}\right|_{0,0} \leqslant C h_{l}^{2 m},
$$

$$
\begin{gathered}
D_{l}=\omega_{l} h_{l}^{-2 m} I, \quad L_{l} \text { and } L_{l}^{*} \text { are diagonally dominant }(c f .[10, p .23]), \\
\left|D_{l}^{-1}\left(R_{l}+S_{l}\right)\right|_{0,0} \leqslant 1, \quad\left|\left(R_{l}+S_{l}\right) D_{l}^{-1}\right|_{0,0} \leqslant 1 .
\end{gathered}
$$

Note that $|\cdot|_{0,0}$ coincides with the usual spectral norm of matrices.

Proof. (a) One verifies that $\widetilde{G}_{l}=S_{l}\left(D_{l}-R_{l}\right)^{-1} \cdot G_{l}^{\nu}$ and $\widetilde{G}_{l}^{\nu}$ have the representations

$$
G_{l}^{\nu}=\left[\begin{array}{cc}
0 & d_{1}^{-1} s\left[d_{2}^{-1} r d_{1}^{-1} s\right]^{\nu-1} \\
0 & {\left[d_{2}^{-1} r d_{1}^{-1} s\right]^{\nu}}
\end{array}\right], \quad \widetilde{G}_{l}^{\nu}=\left[\begin{array}{cc}
{\left[s d_{2}^{-1} r d_{1}^{-1}\right]^{\nu}} & {\left[s d_{2}^{-1} r d_{1}^{-1}\right]^{\nu-1} s d_{2}^{-1}} \\
0 & 0
\end{array}\right]
$$

Assume (4.8a) and let $B$ be as in the proof of Lemma 6. $\left|D_{l}^{1 / 2} G_{l}^{\nu} D_{l}^{-1 / 2}\right|_{0,0}^{2}=$ $\left|D_{l}^{-1 / 2} \widetilde{G}_{l}^{\nu} D_{l}^{1 / 2}\right|_{0,0}^{2}=\left\|B^{2 \nu}+B^{2 \nu-1}\right\| \leqslant 2$ shows $\left|G_{l}^{\nu}\right|_{0,0} \leqslant \sqrt{2}\left|D_{l}^{-1 / 2}\right|_{0,0}\left|D_{l}^{1 / 2}\right|_{0,0}$ $\leqslant C$ and $\left|\widetilde{G}_{l}^{\nu}\right|_{0,0} \leqslant C$. 
(b) Let $\|\cdot\|_{\infty}$ be the matrix norm corresponding to the supremum norm. (4.8b) implies that the $\|\cdot\|_{\infty}$ norm of $D_{l}^{-1}\left(R_{l}+S_{l}\right)=\left(R_{l}+S_{l}\right) D_{l}^{-1}$ and of the adjoint matrix are bounded by 1 . Hence, $(4.8 \mathrm{c})$ holds.

(c) From (4.8c) it follows that $\left\|d_{1}^{-1} s\right\|,\left\|d_{2}^{-1} r\right\|,\left\|s d_{2}^{-1}\right\|,\left\|r d_{1}^{-1}\right\| \leqslant 1(\|\cdot\|$ : spectral norm). Then the representations of $G_{l}^{\nu}$ and $\widetilde{G}_{l}^{\nu}$ yield $\left|G_{l}^{\nu}\right|_{0,0},\left|\widetilde{G}_{l}^{\nu}\right|_{0,0} \leqslant$ $\sqrt{2}$.

We summarize:

Note 6. Assume (4.3) and (4.5). Let the coefficients of $L_{l}$ be sufficiently smooth (cf. Lemma 7). $G_{l}$ is defined by (4.2a), where the splitting (4.2b) is 2-cyclic with $\left|D_{l}^{-1}\right|_{0,0} \leqslant C h_{l}^{2 m}$. Then the smoothing property (2.6) holds for all $\theta, \theta^{\prime} \in$ $[0, m], \theta+\theta^{\prime}=\alpha>0$ with $C_{0}(\nu)$ from $(4.7)$ and $\nu_{\max }(h)=\infty$.

Proof. (4.4a) follows as in Note 5. Thanks to Lemmata 7, 8 the Criterion $3 *$ yields (2.6).

4.4. Perturbations by Lower Order Terms. In the following we shall assume that the difference scheme $L_{l}$ is the sum $L_{l}^{\prime}+L_{l}^{\prime \prime}$, where $L_{l}^{\prime}$ satisfies the smoothing property (2.6). We assume a 2-cyclic splitting of $L_{l}$ and $L_{l}^{\prime}$ :

$$
\begin{gathered}
L_{l}=D_{l}-R_{l}-S_{l}, \quad L_{l}^{\prime}=D_{l}^{\prime}-R_{l}^{\prime}-S_{l}^{\prime}, \\
G_{l}=\left(D_{l}-R_{l}\right)^{-1} S_{l}, \quad G_{l}^{\prime}=\left(D_{l}^{\prime}-R_{l}^{\prime}\right)^{-1} S_{l}^{\prime}, \\
G_{l}^{\prime \prime}=G_{l}-G_{l}^{\prime}, \quad D_{l}^{\prime \prime}=D_{l}-D_{l}^{\prime}, \quad R_{l}^{\prime \prime}=R_{l}-R_{l}^{\prime}, \quad S_{l}^{\prime \prime}=S_{l}-S_{l}^{\prime} .
\end{gathered}
$$

$L_{l}^{\prime \prime}$ is called a lower order term if there is some $\beta>0$ such that

$$
\left|L_{l}^{\prime \prime}\right|_{0,0} \leqslant C h_{l}^{\beta-2 m} \quad(\beta>0, l \geqslant 0) .
$$

The first criterion applies if $\beta>m-\max \left(\theta, \theta^{\prime}\right)$.

CRITERION 4. Let $L_{l}=L_{l}^{\prime}+L_{l}^{\prime \prime}$ and $L_{l}^{\prime}$ have 2-cyclic splittings and define $G_{l}$ and $G_{l}^{\prime}$ by (4.9). Choose the norms by (3.5) and assume

$$
\begin{gathered}
\left|L_{l}^{\prime \prime}\right|_{0, \theta-m} \leqslant C h_{l}^{\beta-m-\theta} \quad\left[\text { or }\left|L_{l}^{\prime \prime}\right|_{m-\theta^{\prime}, 0} \leqslant C h_{l}^{\beta-m-\theta^{\prime}}\right], \\
\left|D_{l}^{\prime-1}\right|_{0,0} \leqslant C h_{l}^{2 m}, \quad\left|L_{l}^{\prime}\right|_{m,-m} \leqslant C
\end{gathered}
$$

and $\beta>m-\theta^{\prime}$ [or $\beta>m-\theta$, respectively]. Then $L_{l}$ has the smoothing property (2.6) if $L_{l}^{\prime}$ has.

For the usual case of $m=1 \beta$ takes the values 1 and 2 . Hence, $\alpha=\theta+\theta^{\prime}>0$ implies $\beta>m-\theta^{\prime}$ or $\beta>m-\theta$. (4.10*) holds if $L_{l}^{\prime \prime}$ is a difference scheme of order $\leqslant 2 m-\beta$ with smooth coefficients (cf. Lemma 7). Note that (4.10*) implies (4.10).

Proof. By (4.10) and (4.11) the estimate $\left|D_{l}^{\prime-1} L_{l}^{\prime \prime}\right|_{0,0} \leqslant C h_{l}^{\beta}$ holds. The same norm of $D_{l}^{\prime-1} D_{l}^{\prime}, D_{l}^{\prime-1} R_{l}^{\prime \prime}$, and $D_{l}^{\prime-1} S_{l}^{\prime \prime}$ is also of order $O\left(h_{l}^{\beta}\right)$ since the splitting is 2-cyclic. Hence, $\left|G_{l}^{\prime \prime}\right|_{0,0} \leqslant C h_{l}^{\beta}$ is valid for sufficiently small $h_{l}$. The second estimate of (4.11) implies $\left|L_{l}^{\prime}\right|_{0,0} \leqslant C h_{l}^{-2 m}$. Thus, $\left|G_{l}^{\prime}\right|_{0,0} \leqslant C$ holds, too. $X(\nu)=G_{l}^{\nu}-G_{l}^{\prime \nu}$ 
can be estimated by

$$
\begin{aligned}
|X(\nu)|_{m-\theta^{\prime}, 0} & \leqslant|X(\nu)|_{0,0} \leqslant c\left(\nu, h_{l}\right):=\sum_{\mu=1}^{\nu}\left(\begin{array}{l}
\nu \\
\mu
\end{array}\right)\left|G_{l}^{\prime}\right|_{0,0}^{\mu}\left|G_{l}^{\prime \prime}\right|_{0,0}^{\nu-\mu} \\
& \leqslant \sum_{\mu=1}^{\nu}\left(\begin{array}{l}
\nu \\
\mu
\end{array}\right) C^{\mu}\left[C h_{l}^{\beta}\right]^{\nu-\mu}=C^{\nu}\left[\left(1+h_{l}^{\beta}\right)^{\nu}-1\right] .
\end{aligned}
$$

The further terms of

$$
\left\|L_{l} G_{l}^{\nu}\right\|_{2,1} \leqslant\left\|L_{l}^{\prime} G_{l}^{\prime \nu}\right\|_{2,1}+\left|L_{l}^{\prime \prime}\right|_{0, \theta-m}\left|G_{l}^{\nu}\right|_{m-\theta^{\prime}, 0}+\left|L_{l}^{\prime}\right|_{0, \theta-m}|X(\nu)|_{m-\theta^{\prime}, 0}
$$

are bounded by

$$
\left|L_{l}^{\prime \prime}\right|_{0, \theta-m} \leqslant C h_{l}^{\beta-m-\theta}, \quad\left|L_{l}^{\prime}\right|_{0, \theta-m} \leqslant C h_{l}^{-m-\theta}, \quad\left|G_{l}^{\nu}\right|_{m-\theta^{\prime}, 0} \leqslant C^{\nu} .
$$

Since $L_{l}^{\prime}$ satisfies (2.6) with $C_{0}^{\prime}(\nu)$ and $\nu_{\max }^{\prime}(h)$ one obtains $\left\|L_{l} G_{l}^{\nu}\right\|_{2,1} \leqslant h_{l}^{-\alpha} c_{0}\left(\nu, h_{l}\right)$ with $c_{0}(\nu, h)=C_{0}^{\prime}(\nu)+h^{\theta^{\prime}-m+\beta} C^{\nu}+C h^{\theta^{\prime}-m} c(\nu, h) . \theta^{\prime}-m+\beta>0$ implies $c_{0}(\nu, 0)$ $=C_{0}^{\prime}(\nu)$. Thus, there exists $\nu_{\max }(h) \leqslant \nu_{\text {max }}^{\prime}(h)$ with $\nu_{\max }(0)=\infty$ such that $c_{0}(\nu, h)$ $\leqslant C_{0}(\nu):=2 C_{0}^{\prime}(\nu)$ for all $0 \leqslant \nu \leqslant \nu_{\max }(h)$. In the case of the second inequality of $\left(4.10^{*}\right)$ and $\beta-m<\theta$ apply the analogous estimates to $\widetilde{G}_{l}^{\nu} L_{l}=L_{l} G_{l}^{\nu}$.

The following criterion is applicable for all $\beta>0$. On the other hand $L_{l}^{\prime}$ must satisfy not only the smoothing property but also the sufficient conditions of Criterion $3^{*}$.

CRITERION 5. Let $L_{l}=L_{l}^{\prime}+L_{l}^{\prime \prime}$ and $L_{l}^{\prime}$ have 2-cyclic splittings and define $G_{l}$ and $G_{l}^{\prime}$ by (4.9). Assume (4.3), (4.10), (4.11), and $\left|L_{l}^{\prime \prime}\right|_{2 m, 0} \leqslant C,\left|L_{l}^{\prime *}\right|_{2 m, 0} \leqslant C$. Moreover, the estimates (4.4a) and $\left(4.4 \mathrm{~b}^{*}\right)$ must be valid for $L_{l}^{\prime}, G_{l}^{\prime}, \widetilde{G}_{l}^{\prime}$ (instead of $\left.L_{l}, G_{l}, \widetilde{G}_{l}\right)$. Then the smoothing property (2.6) holds for $L_{l}$, too.

Proof. Repeating the proof of Criterion. 4 for the special case of $\theta=\theta^{\prime}=m$ one obtains (4.4a). The same proof shows $\left(4.4 b^{*}\right)$ for a suitable choice of $\nu_{\max }(h)$. Hence Criterion $3^{*}$ implies (2.6).

Note 6 and Criterion 5 establish the following result.

Note 7. Assume (4.3) and $C^{-1}|u|_{m}^{2} \leqslant \operatorname{Re}\left(L_{l} u, u\right)+\lambda_{0}|u|_{0}^{2} \leqslant C|u|_{m}^{2}$ for some real $\lambda_{0}\left(H_{0}^{m}\right.$-coerciveness of $\left.L_{l}\right) . L_{l}$ must consist of the terms $T^{\gamma} \partial^{\beta} c\left(x, h_{l}\right) \partial^{\beta^{\prime}} d e$ scribed in Lemma 7. $G_{l}$ is defined by (4.2a), where the splitting (4.2b) is 2-cyclic with $\left|D_{l}^{-1}\right|_{0,0} \leqslant C h_{l}^{2 m}$. Then the smoothing property (2.6) holds with $C_{0}(\nu)$ from (4.7).

Proof. Define $L_{l}^{\prime}=\left(L_{l}+L_{l}^{*}\right) / 2+\lambda_{0} I$ and $L_{l}^{\prime \prime}=L_{l}-L_{l}^{\prime} .\left|L_{l}^{\prime \prime}\right|_{0,0} \leqslant C h_{l}^{2 m-1}$ and $\left|D_{l}^{-1}\right|_{0,0} \leqslant C h_{l}^{2 m}$ imply $\left|D_{l}^{\prime-1}\right|_{0,0} \leqslant C^{\prime} h_{l}^{2 m}$ for sufficiently small $h_{l}$. Hence, Note 6 shows that $(4.4 \mathrm{a})$ and $\left(4.4 \mathrm{~b}^{*}\right)$ hold for $L_{l}^{\prime}$ and $G_{l}^{\prime}$. (2.6) follows by Criterion 5 .

4.5. Perturbation at the Boundary. In particular, if special discretizations are used at points near the boundary, the difference scheme $L_{l}$ is a sum of a scheme $L_{l}^{\prime}$ with smooth coefficients as studied in the foregoing section and a further term $L_{l}^{\prime \prime}$ with $\left(L_{l}^{\prime \prime} u\right)(x) \neq 0$ only at points near the boundary. The following note shows the smoothing property for an important class of discretizations.

Note 8. Let $L_{l}=L_{l}^{\prime}+L_{l}^{\prime \prime}$ and $L_{l}^{\prime}$ have 2-cyclic splittings with diagonal matrices $D_{l}, D_{l}^{\prime}$ and define $G_{l}$ and $G_{l}^{\prime}$ by (4.9). If $\left(L_{l}^{\prime \prime} u\right)(x) \neq 0$ for some $u,\left|x-x^{\prime}\right| \leqslant C h_{l}$ must hold for some $x^{\prime}=\nu^{\prime} h_{l} \notin \Omega\left(h_{l}\right)\left(c f\right.$. Lemma 2). Moreover, $\left(L_{l} u\right)(x)$ and 
$\left(L_{l}^{\prime} u\right)(x)$ must depend only on $u\left(x^{\prime}\right)$ with $\left|x^{\prime}-x\right| \leqslant C h_{l}\left(x, x^{\prime} \in \Omega\left(h_{l}\right)\right)$. A ssume that (4.4a) and $\left(4.4 \mathrm{~b}^{*}\right)$ hold for $L_{l}^{\prime}, G_{l}^{\prime}$, and $\widetilde{G}_{l}^{\prime}$ with $C(\nu)$ from (4.7) (sufficient conditions are those of Note 7). Furthermore, (4.3) and (3.6b) with some $\vartheta \in(0,1 / 2)$ are required for $L_{l}^{\prime}$ (instead of $L_{l}$ ). Let

$$
\begin{gathered}
\left|D_{l}^{\prime-1}\right|_{0,0} \leqslant C h_{l}^{2 m}, \quad\left|R_{l}+S_{l}\right|_{0,0} \leqslant C h_{l}^{-2 m}, \\
\left|D_{l}^{\prime-1} L_{l}^{\prime}\right|_{0,0} \leqslant C, \quad\left|L_{l}^{\prime} D_{l}^{\prime-1}\right|_{0,0} \leqslant C .
\end{gathered}
$$

The inequalities

(4.12b) $0 \leqslant D_{l}^{-1}\left(R_{l}+S_{l}\right) \leqslant D_{l}^{\prime-1}\left(R_{l}^{\prime}+S_{l}^{\prime}\right), \quad 0 \leqslant\left(R_{l}+S_{l}\right) D_{l}^{-1} \leqslant\left(R_{l}^{\prime}+S_{l}^{\prime}\right) D_{l}^{\prime-1}$

must hold for all entries of the matrices. Then the smoothing property (2.6) is valid with the same $\nu_{\max }(h)$ as for $L_{l}^{\prime}$.

It is to be emphasized that $D_{l}$ is not required to be uniformly bounded.

Proof. (1) We abbreviate $|\cdot|_{0,0}$ by $\|\cdot\|$. There is $\gamma$ as in Lemma 2 such that

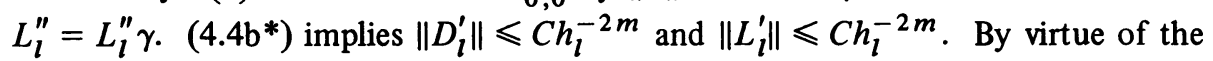
Perron-Frobenius theory (cf. [10, p. 26]) $\left\|D_{l}^{-1} L_{l}\right\| \leqslant\left\|D_{l}^{\prime-1} L_{l}^{\prime}\right\| \leqslant C$ can be concluded from $(4.12 \mathrm{a}, \mathrm{b})$. Therefore,

$$
\begin{aligned}
\left|D_{l}^{\prime} D_{l}^{-1} L_{l}\right|_{2 m, 0} & \leqslant\left|D_{l}^{\prime} D_{l}^{-1} L_{l}-L_{l}^{\prime}\right|_{2 m, 0}+\left|L_{l}^{\prime}\right|_{2 m, 0} \leqslant\left|\left(D_{l}^{\prime} D_{l}^{-1} L_{l}-L_{l}^{\prime}\right) \gamma\right|_{2 m, 0}+C \\
& \leqslant\left(\left\|D_{l}^{\prime}\right\|\left\|D_{l}^{-1} L_{l}\right\|+\left\|L_{l}^{\prime}\right\|\right)|\gamma|_{2 m, 0}+C \leqslant C^{\prime}
\end{aligned}
$$

yields the first inequality of $(4.13 a)$ :

$$
\left|D_{l}^{\prime} D_{l}^{-1} L_{l}\right|_{2 m, 0} \leqslant C, \quad\left|D_{l}^{\prime *} D_{l}^{*-1} L_{l}^{*}\right|_{2 m, 0} \leqslant C
$$

Similarly, the second estimate is proved.

(2) Let $d_{1}, d_{2}, r$, and $s$ be as in the proof of Lemma 6. (4.12b) yields $0 \leqslant$ $d_{1}^{-1} s \leqslant d_{1}^{\prime-1} s^{\prime}$, etc. Hence

$$
0 \leqslant G_{l}^{\nu} \leqslant G_{l}^{\prime \nu}, \quad 0 \leqslant \widetilde{G}_{l}^{\nu} \leqslant \widetilde{G}_{l}^{\nu} \quad(\nu \geqslant 0)
$$

follows. The Perron-Frobenius theory shows $\left\|G_{l}^{\nu}\right\| \leqslant\left\|G_{l}^{\prime \nu}\right\|$. By $\left\|D_{l} G_{l}^{\nu}\right\| \leqslant$ $\|s\|\left(1+\left\|r d_{1}^{-1}\right\|\right)\left\|G_{l}^{\nu-1}\right\| \leqslant C h_{l}^{-2 m}$ [cf. (4.12a), $\left.\left(4.4 \mathrm{~b}^{*}\right)\right]$ and $\left\|D_{l}^{\prime-1}\right\| \leqslant C h_{l}^{2 m}$ we obtain the first estimate of $(4.13 \mathrm{c})$ :

$$
\left\|D_{l}^{\prime-1} D_{l} G_{l}^{\nu}\right\| \leqslant C, \quad\left\|\widetilde{G}_{l}^{\nu} D_{l} D_{l}^{\prime-1}\right\| \leqslant C \quad\left(1 \leqslant \nu \leqslant \nu_{\max }\left(h_{1}\right)\right) .
$$

The proof of the second one is similar.

(3) (4.13a) and (4.13b) imply

$$
\left|L_{l} G_{l}^{\nu}\right|_{0,-2 m} \leqslant C, \quad\left|L_{l} G_{l}^{\nu}\right|_{2 m, 0} \leqslant C \quad\left(1 \leqslant \nu \leqslant \nu_{\max }\left(h_{1}\right)\right) .
$$

E.g., the first inequality follows from

$$
\begin{aligned}
\left|L_{l} G_{l}^{\nu}\right|_{0,-2 m} & \leqslant\left|L_{l} D_{l}^{-1} D_{l}^{\prime}\right|_{0,-2 m}\left|D_{l}^{\prime-1} D_{l} G_{l}^{\nu}\right|_{0,0} \\
& \leqslant\left|D_{l}^{\prime *} D_{l}^{*-1} L_{l}^{*}\right|_{2 m, 0}\left\|D_{l}^{\prime-1} D_{l} G_{l}^{\nu}\right\| \leqslant C .
\end{aligned}
$$


(4) Let $\gamma$ be as in Lemma 2. By (3.6b) $\left|L_{l}^{\prime-1}\right|_{\vartheta-m, \vartheta+m} \leqslant C$ holds for some $0<\vartheta<1 / 2$. Lemma 2 proves $|\gamma|_{\vartheta+m, 0} \leqslant C h_{l}^{\vartheta+m}$. Interpolation of (4.4a) and $\left(4.4 \mathrm{~b}^{*}\right)$ yields $\left|L_{l}^{\prime} G_{l}^{\prime \nu}\right|_{0, \vartheta-m} \leqslant C h_{l}^{-m-\vartheta} /(\nu+1)^{\beta}$ with $\beta=(m+\vartheta) /(2 m)$. Therefore,

$$
\left\|\gamma G_{l}^{\prime \nu}\right\| \leqslant|\gamma|_{\vartheta+m, 0}\left|L_{l}^{\prime-1}\right|_{\vartheta-m, \vartheta+m}\left|L_{l}^{\prime} G_{l}^{\prime \nu}\right|_{0, \vartheta-m} \leqslant C /(\nu+1)^{\beta}
$$

is valid. Applying again the Perron-Frobenius theory, we obtain

$$
\left\|\gamma G_{l}^{\nu}\right\| \leqslant C /(\nu+1)^{\beta}, \quad \beta=(m+\vartheta) /(2 m) \quad\left(0 \leqslant \nu \leqslant \nu_{\max }\left(h_{1}\right)\right)
$$

from (4.13b). Now,

$$
\left\|L_{l}^{\prime \prime} G_{l}^{\nu}\right\| \leqslant C h_{l}^{-2 m} /(\nu+1)^{\beta}, \quad \beta=(m+\vartheta) /(2 m) \quad\left(1 \leqslant \nu \leqslant \nu_{\max }\left(h_{1}\right)\right)
$$

can be concluded from $\left\|L_{l}^{\prime \prime} G_{l}^{\nu}\right\|=\left\|L_{l}^{\prime \prime} G_{l}\right\|\left\|\gamma G_{l}^{\nu-1}\right\|$, since there is $\gamma$ satisfying the conditions of Lemma 2 with $L_{l}^{\prime \prime} G_{l}=L_{l}^{\prime \prime} G_{l} \gamma$. The second term is estimated in (4.13e). Split the first term into $L_{l} G_{l}-L_{l}^{\prime} G_{l}$. $\left\|L_{l} G_{l}\right\| \leqslant C h_{l}^{-2 m}\left|L_{l} G_{l}\right|_{0,-2 m} \leqslant C h_{l}^{-2 m}$ follows "from (4.13d) $(\nu=1)$. (4.4b*) for $L_{l}^{\prime}$ and (4.13b) yield $\left\|L_{l}^{\prime} G_{l}\right\| \leqslant C h_{l}^{-2 m}$.

(5) Using $L_{l}^{\prime}\left(G_{l}^{\prime \nu}-G_{l}^{\nu}\right)=-\Sigma_{\mu=0}^{\nu-1} L_{l}^{\prime} G_{l}^{\prime \mu} G_{l}^{\prime \prime} G_{l}^{\nu-\mu-1}$ and $G_{l}^{\prime \prime}=G_{l}^{\prime \prime} \gamma$, one obtains

$$
\begin{aligned}
\left\|L_{l}^{\prime}\left(G_{l}^{\prime \nu}-G_{l}^{\nu}\right)\right\| & \leqslant \sum_{\mu=0}^{\nu-1}\left\|L_{l}^{\prime} G_{l}^{\prime \mu}\right\|\left\|G_{l}^{\prime \prime}\right\|\left\|\gamma G_{l}^{\nu-\mu-1}\right\| \\
& \leqslant h_{l}^{-2 m} C^{\prime} \sum_{\mu=0}^{\nu-1}\left[(\mu+1)^{-1}(\nu-\mu)^{-\beta}\right] \leqslant C h_{l}^{-2 m} /(\nu+1)^{\vartheta / m}
\end{aligned}
$$

This estimate, (4.4a) (for $\left.L_{l}^{\prime}\right)$, and (4.13f) yield (4.4a) for $L_{l}$ :

$$
\begin{aligned}
\left\|L_{l} G_{l}^{\nu}\right\| \leqslant\left\|L_{l}^{\prime \prime} G_{l}^{\nu}\right\|+\left\|L_{l}^{\prime}\left(G_{l}^{\nu}-G_{l}^{\prime \nu}\right)\right\|+\left\|L_{l}^{\prime} G_{l}^{\prime \nu}\right\| \leqslant C h_{l}^{-2 m} /(\nu+1)^{\vartheta / m} & \left(1 \leqslant \nu \leqslant \nu_{\max }\left(h_{1}\right)\right) .
\end{aligned}
$$

Repeating the proof of Criterion $3^{*}$ yields (2.6).

Example. Consider the Shortley-Weller discretization $L_{l}$ (cf. last example of Section 3). $L_{l}^{\prime}$ is the usual five-point formula. Hence, $(4.4 \mathrm{a})$ and $\left(4.4 \mathrm{~b}^{*}\right)$ are fulfilled with $\nu_{\max }(h)=\infty$. (3.6b) holds for all $\theta_{0}=\theta_{0}^{\prime}<1 / 2$. Also the conditions $(4.12 \mathrm{a}, \mathrm{b})$ are satisfied. Thus, the smoothing property holds for all $\nu\left(\nu_{\max }=\infty\right)$.

Mathematisches Institut

Universität zu Köln

Weyertal 86-90

D-5000 Köln 41, Federal Republic of Germany

1. G. P. ASTRACHANCEV, "An iterative method of solving elliptic net problems," $\check{Z}$. Vyčish. Mat. i Mat. Fiz., v. 11, 1971, pp. 439-448.

2. N. S. BACHVALOV, "On the convergence of a relaxation method with natural constraints on the elliptic operator," Ž. Vycisl. Mat. $i$ Mat. Fiz., v. 6, 1966, pp. 861-885.

3. A. BRANDT, "Multi-level adaptive solutions to boundary-value problems," Math. Comp., v. 31, 1977, pp. 333-390.

4. R. P. FEDORENKO, "The speed of convergence of one iterative process," ž. Vyčisl. Mat. i Mat. Fiz., v. 4, 1964, pp. 559-564. 
5. W. HACKBUSCH, "On the multi-grid method applied to difference equations," Computing, v. 20, 1978, pp. 291-306.

6. W. HACKBUSCH, "On the convergence of multi-grid iterations," Beiträge Numer. Math., v. 9. (To appear.)

7. W. HACKBUSCH, “On the regularity of difference schemes." (To appear.)

8. TH. MEIS \& U. MARCOWIT Z, Numerische Behandlung partieller Differentialgleichungen, Springer-Verlag, Berlin, Heidelberg, New York, 1978.

9. R. A. NICOLAIDES, "On the $l^{2}$ convergence of an algorithm for solving finite element equations," Math. Comp., v. 31, 1977, pp. 892-906.

10. R. S. VARGA, Matrix Iterative Analysis, Prentice-Hall, Englewood Cliffs, N.J., 1962.

11. P. WESSELING, A Convergence Proof for a Multiple Grid Method, Delft University of Technology, Report NA-21, 1978.

12. W. HACKBUSCH, "Bemerkungen zur iterierten Defektkorrektur und zu ihrer Kombination mit Mehrgitterverfahren, Rev. Roumaine Math. Pures Appl. (To appear.) 